\title{
EDUCAÇÃO ALIMENTAR E NUTRICIONAL: Análise de Ações com Adultos a Partir do Marco de Referência
}

\author{
Lisiane Giusti ${ }^{1}$ \\ Ana Luiza Sander Scarparo 2 \\ Eliziane Nicolodi Francescato Ruiz ${ }^{3}$
}

\begin{abstract}
RESUMO
No presente estudo objetivou-se revisar e analisar produções brasileiras sobre intervenções de Educação Alimentar e Nutricional com adultos após a criação do Marco de Referência de Educação Alimentar e Nutricional para Políticas Públicas. Realizou-se uma revisão da produção científica, considerando como critérios de elegibilidade: intervenções de educação alimentar e nutricional, público-alvo de adultos e ações/intervenções realizadas entre o período de 2012 e 2018. Para a análise foram utilizados os seguintes elementos: o objetivo das ações de EAN, o público-alvo, a metodologia utilizada e os principais resultados dos estudos, a presença dos princípios do Marco de EAN nas intervenções realizadas nos trabalhos e uso de abordagens e recursos educacionais problematizadores e ativos que favorecessem o diálogo entre os participantes. Dos 82 artigos encontrados foram selecionados 10, que responderam aos critérios de elegibilidade. Identificou-se que 9 dos estudos apresentaram uma abordagem relacionada a um ou mais dos princípios do Marco. Nenhum dos trabalhos analisados, porém, utilizou diretamente o Marco de EAN como referência na descrição metodológica ou utilizou-o como base teórica. E dos estudos analisados, somente 4 realizaram as ações com o uso de abordagens e recursos educacionais problematizadores e ativos que favorecessem o diálogo entre os participantes. Assim, destaca-se a necessidade de construção de novas perspectivas para as práticas de educação alimentar e nutricional e a importância de aperfeiçoar os estudos com a utilização do Marco como referência.
\end{abstract}

Palavras-chave: educação alimentar e nutricional; marco de referência de EAN; ações educativas; adultos.

FOOD AND NUTRITION EDUCATION: ANALYSIS OF ACTIONS WITH ADULTS BASED ON THE MARCO DE REFERÊNCIA ABSTRACT

The present work is aimed to review and analyze the brazilian production on Food and Nutrition Education interventions with adults after the creation of Marco de Referência de Educação Alimentar e Nutricional para Políticas Públicas [Reference Landmark of Food and Nutrition Education]. A review of the scientific production was carried out considering as eligibility criteria: food and nutritional education interventions, target public of adults and actions / interventions carried out between the period of 2012 to 2018. For the analysis the following elements were used: the objective EAN actions, the target audience, the methodology used and the main results of the studies, which of the principles of the EAN landmark were present in the interventions carried out in the studies and use of problematizing and active educational approaches and resources that favored dialogue between the participants. We found 82 articles and 10 articles were selected that met the eligibility criteria. It was identified that, with $90 \%$ of the studies presented an approach related to one or more of the principles of the landmark. However, none of the studies analyzed directly used the EAN landmark as a reference in the methodological description or used it as a theoretical basis in the construction of the text. In addition, $40 \%$ of the studies analyzed carried out actions with the use of problematizing and active educational approaches and resources that favored dialogue among the participants. With this, it is necessary to construct new perspectives for the practices of food and nutritional education and also to improve the studies by using the landmark as reference.

Keywords: food and nutrition education; Ean reference landmark; education actions; adult.

Recebido em: 9/8/2019

Aceito em: 20/10/2019

\footnotetext{
Universidade Federal do Rio Grande do Sul - UFRGS. Porto Alegre/RS, Brasil. http://lattes.cnpq.br/1305430326260359. https://orcid. org/0000-0003-1053-6717.

2 Universidade Federal do Rio Grande do Sul - UFRGS. Programa de Pós-Graduação em Educação em Ciências: Química da Vida e Saúde. Porto Alegre/RS, Brasil. http://lattes.cnpq.br/0496384259988832. https://orcid.org/0000-0001-6514-8549.

3 Autora correspondente. Universidade Federal do Rio Grande do Sul, Faculdade de Medicina. Av. João Pessoa, 31 - Santa Cecília. Porto Alegre/ RS, Brasil. CEP 90040-000. http://lattes.cnpq.br/6460045934453096. https://orcid.org/0000-0002-8632-6612. elizianeruiz@yahoo.com.br
} 


\section{INTRODUÇÃO}

Nas últimas décadas mudanças sociais, políticas, econômicas e culturais têm influenciado o estilo de vida da população brasileira, resultando em modificações no padrão de saúde e no consumo alimentar, as quais impactaram, significativamente, o seu estado nutricional. Observa-se um aumento contínuo nos índices de sobrepeso e obesidade em todas as faixas etárias e camadas da sociedade, o que acarreta novos problemas de saúde pública, muitos deles relacionados à alimentação e à nutrição (BRASIL, 2012a, 2014a). Segundo dados do Ministério da Saúde (BRASIL, 2018), mais da metade da população, 55,7\%, apresentam excesso de peso.

Articular ações para o enfrentamento do sobrepeso, da obesidade e das doenças decorrentes da má alimentação consiste em uma das diretrizes do Pacto para a Promoção da Alimentação Saudável, lançado, em 2015, por meio do Decreto no 8.553/2015 (BRASIL, 2015). O documento tem como finalidade ampliar as condições de oferta, disponibilidade e consumo de alimentos saudáveis e combater as doenças associadas à má alimentação. Para tanto, os eixos de suas ações consistem em aumentar a oferta e a disponibilidade de alimentos saudáveis, reduzir o uso de agrotóxicos, diminuir os teores de sal, açúcar e gordura dos alimentos industrializados, aumentar o consumo de alimentos da agricultura familiar e fomentar a Educação Alimentar e Nutricional (EAN) nos serviços de saúde, de educação e de assistência social.

Mesmo que a promoção de práticas alimentares saudáveis seja, atualmente, prioridade das políticas públicas de saúde, alimentação e nutrição do país, ainda não se conseguiu alcançar resultados satisfatórios na prevenção e no controle da obesidade (PEREIRA et al., 2015). O enfrentamento dessa situação requer que o Estado adote medidas e ações em todos os setores, com o apoio e a participação da sociedade civil (BRASIL, 2014a).

Em 2012 foi publicado o Marco de Referência de Educação Alimentar e Nutricional para as Políticas Públicas, tendo como objetivo promover um campo comum de reflexão e orientação da prática no conjunto de iniciativas de EAN que tenham origem, principalmente, na ação pública, e que contemplem os diversos setores vinculados ao processo de produção, distribuição, abastecimento e consumo de alimentos (BRASIL, 2012b).

Segundo o Marco de Referência, a adoção do conceito "Educação Alimentar e Nutricional" compreende as questões relacionadas ao alimento, à alimentação, à produção, ao abastecimento e aos aspectos nutricionais. A EAN, portanto, foi definida como:

Educação Alimentar e Nutricional, no contexto da realização do Direito Humano à Alimentação Adequada e da garantia da Segurança Alimentar e Nutricional, é um campo de conhecimento e de prática contínua e permanente, transdisciplinar, intersetorial e multiprofissional que visa promover a prática autônoma e voluntária de hábitos alimentares saudáveis (BRASIL, 2012b, p. 23).

Além disso, de acordo com o proposto no documento, a prática da EAN deve fazer uso de abordagens e recursos educacionais problematizadores e ativos que favoreçam o diálogo junto a indivíduos e grupos populacionais, além de considerar as fases da vida, as etapas do sistema alimentar e as interações e significados que compõem o comportamento alimentar (BRASIL, 2012b). 
Essa compreensão da necessidade da abordagem da EAN, para além da transmissão de informações sobre alimentação e nutrição, corrobora os pressupostos de um modelo epistemológico construtivista, conforme proposto por Piaget, que fundamenta uma pedagogia relacional (SCARPARO, 2017). Nesse sentido, para a realização de uma ação de EAN, é importante problematizar com o grupo a realidade vivida, incentivando o diálogo e a reflexão crítica, exercitando o questionamento e a busca de soluções a partir das dificuldades identificadas (RAMOS; SCARPARO, 2016)

O Marco de Referência de EAN (BRASIL, 2012b) propõe nove princípios para a realização das ações de EAN: 1 - sustentabilidade social, ambiental e econômica, envolvida desde a produção até o consumo dos alimentos; 2 - abordagem do sistema alimentar, na sua integridade, a fim de favorecer que o indivíduo faça escolhas alimentares mais conscientes; 3 - valorização da cultura alimentar local e respeito à diversidade de opiniões e perspectivas, considerando a legitimidade dos saberes de diferentes naturezas; 4 - a comida e o alimento como referências, valorizando a culinária como prática emancipatória, pois as pessoas não se alimentam de nutrientes, mas de alimentos e suas combinações; 5 - a promoção do autocuidado e da autonomia, fazendo com que as pessoas se tornem agentes promotores de sua saúde; 6 - a educação como processo permanente e gerador de autonomia e participação ativa e informada dos sujeitos; 7 - a diversidade nos cenários de prática, considerando os diversos espaços sociais e os diferentes grupos populacionais; 8 - intersetorialidade, com articulação dos distintos setores governamentais; 9 - planejamento, avaliação e monitoramento das ações.

Cervato-Mancuso et al. (2016), na revisão de estudos realizados entre os anos de 2000 e 2012, constataram que o intuito das intervenções de EAN vem se modificando. Antigamente, a EAN estava relacionada à distribuição de alimentos e ao aumento do conhecimento sobre alimentação, centrando-se nas necessidades biológicas dos indivíduos. Atualmente o foco dos trabalhos é a mudança de comportamento alimentar individual, compreendendo-o como um resultado de relações sociais e culturais. Santos (2012), entretanto, aponta que, mesmo com a crescente valorização da EAN nos últimos anos, há dificuldade no desenvolvimento das ações, em virtude da escassez de referencial teórico, metodológico e operacional.

Diante desse contexto, observa-se uma crescente demanda para a realização de ações de EAN e, consequentemente, um aumento na produção de documentos contendo tanto estratégias educativas para mudança das práticas alimentares e da situação nutricional da população quanto de orientações para o desenvolvimento dessas ações. Um desses documentos é o Marco de Referência de EAN. Nesse sentido, verifica-se a pertinência de investigar se o preconizado pelo Marco está sendo utilizado no planejamento e desenvolvimento das ações de EAN.

O objetivo do presente estudo consiste em analisar a atual produção científica, no Brasil, sobre ações de Educação Alimentar e Nutricional com adultos, após a criação do Marco de Referência de EAN para Políticas Públicas. Além disso, procura-se verificar quais dos princípios do Marco estão presentes nas intervenções e constatar se tais ações fazem uso de abordagens e recursos educacionais problematizadores e ativos que favoreçam o diálogo entre os participantes. 


\section{METODOLOGIA DA PESQUISA}

Trata-se de uma revisão da literatura sobre estudos com intervenção de EAN. Os estudos foram identificados na base Portal Periódicos Capes (Coordenação de Aperfeiçoamento de Pessoal de Nível Superior). A busca ocorreu a partir dos seguintes passos: - descritor: "Educação alimentar e nutricional" (descritor entre aspas, pois esta é a expressão utilizada no Marco de Referência em EAN); - tipo de recurso: artigos - data: entre 2012 e 2018 (a busca foi realizada em 5/6/2018); - idioma: português (em razão de o Marco de EAN ser um documento oficial do Brasil); - publicações revisadas por pares.

Encontrou-se um total de 82 estudos, os quais foram submetidos a um processo de triagem, realizado por meio da leitura de títulos e resumos, que buscou apenas trabalhos não duplicados com as seguintes características: intervenções de educação alimentar e nutricional, o público-alvo de adultos, ações/intervenções realizadas entre o período de 2012 e 2018. Foram, então, selecionados, para a leitura completa do texto, 11 artigos.

Após, foi realizada a leitura completa dos textos, excluindo-se um artigo cuja intervenção de EAN ocorreu antes do período selecionado. A Figura 1 apresenta o fluxograma da seleção dos artigos.

Figura 1 - Fluxograma das fases de identificação, triagem e seleção de artigos sobre intervenções de educação alimentar e nutricional

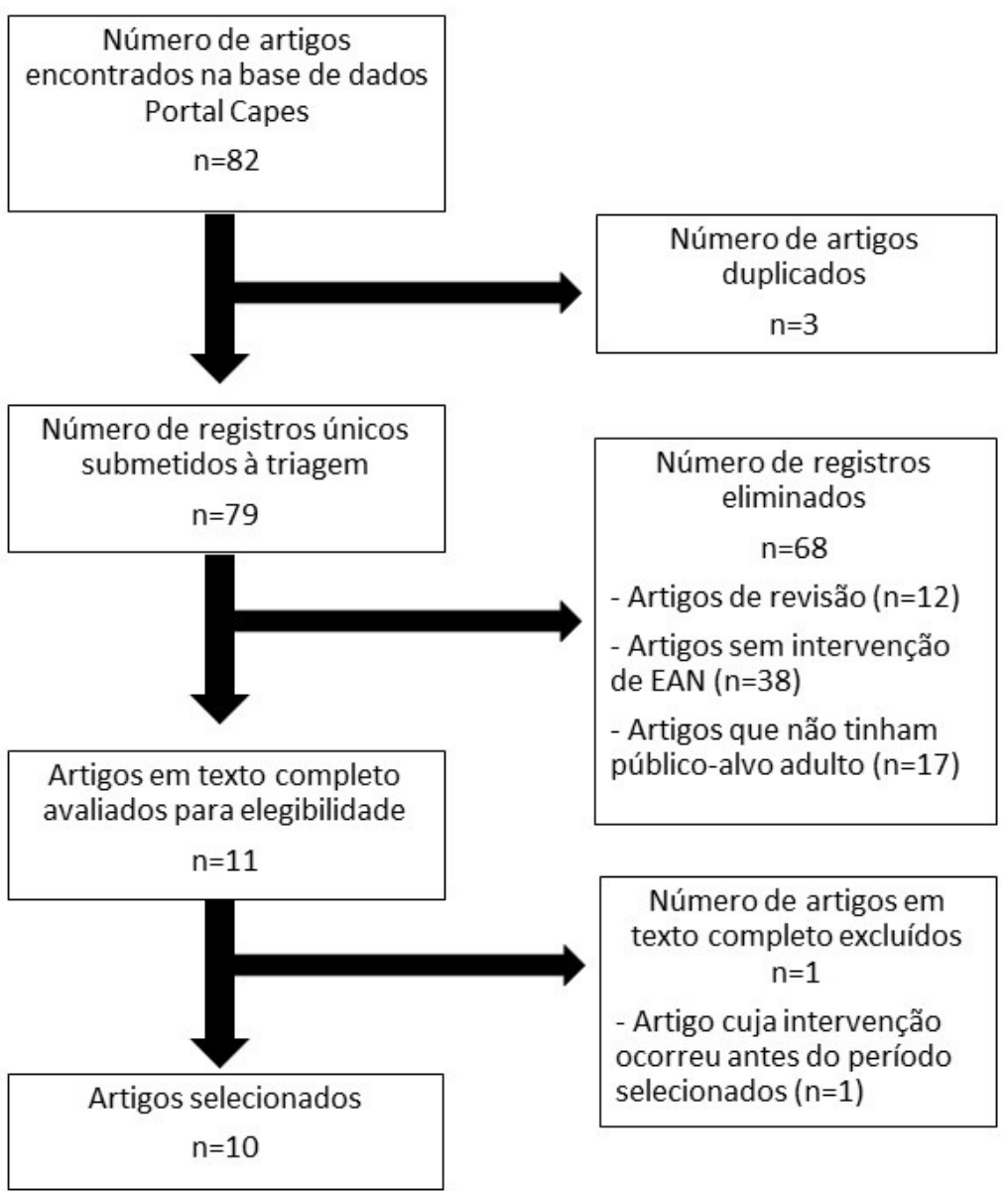

Fonte: Elaboração própria. 


\section{RESULTADOS}

Foram analisados 10 estudos que apresentaram intervenção de Educação Alimentar e Nutricional, de acordo com os critérios de inclusão. A Tabela 1 apresenta uma sistematização dos artigos quanto aos objetivos do estudo, ao público-alvo, ao período de realização ou duração, à metodologia utilizada na intervenção, ao procedimento de avaliação e aos resultados encontrados.

Tabela 1 - Descritivo das ações de EAN, o público-alvo, a metodologia utilizada e os principais resultados dos estudos

\begin{tabular}{|c|c|c|c|c|c|c|}
\hline Autor & Objetivo & Público-Alvo & Período & Intervenção realizada & $\begin{array}{l}\text { Avaliação da } \\
\text { intervenção }\end{array}$ & $\begin{array}{l}\text { Resultados } \\
\text { encontrados }\end{array}$ \\
\hline $\begin{array}{l}\text { Beledelli; } \\
\text { Santolin, } \\
2017\end{array}$ & $\begin{array}{c}\text { Avaliar o perfil } \\
\text { nutricional antes } \\
\text { e após a aplicação } \\
\text { dos Dez Passos } \\
\text { da Alimentação } \\
\text { Saudável e Dez Passos } \\
\text { da Alimentação } \\
\text { Adequada e Saudável. }\end{array}$ & $\begin{array}{l}\text { Grupo de } 22 \\
\text { praticantes de } \\
\text { Pilates em uma } \\
\text { academia do } \\
\text { Norte do Rio } \\
\text { Grande do Sul. }\end{array}$ & $\begin{array}{c}\text { Janeiro a abril } \\
\text { de } 2016 . \\
\text { (4 meses) }\end{array}$ & $\begin{array}{l}\text { Aplicação de orientações } \\
\text { dos Dez Passos da } \\
\text { Alimentação Saudável (no } \\
\text { grupo 1) e Dez Passos da } \\
\text { Alimentação Adequada e } \\
\text { Saudável (no grupo 2). }\end{array}$ & $\begin{array}{l}\text { Foi realizada avaliação } \\
\text { antropométrica antes } \\
\text { e após a intervenção. }\end{array}$ & $\begin{array}{c}\text { Participantes } \\
\text { apresentaram } \\
\text { melhores resultados } \\
\text { na avaliação } \\
\text { antropométrica. }\end{array}$ \\
\hline $\begin{array}{l}\text { Brito et al., } \\
2017\end{array}$ & $\begin{array}{l}\text { Relatar a experiência } \\
\text { da implantação } \\
\text { de práticas de } \\
\text { educação alimentar e } \\
\text { nutricional, em grupo, } \\
\text { para pessoas com } \\
\text { doenças infecciosas } \\
\text { do Instituto Nacional } \\
\text { de Infectologia } \\
\text { Evandro Chagas-RJ. }\end{array}$ & $\begin{array}{l}12 \text { indivíduos } \\
\text { portadores } \\
\text { de doenças } \\
\text { infecciosas e } \\
\text { parasitárias com } \\
\text { diagnósticos de } \\
\text { excesso de peso } \\
\text { e de síndrome } \\
\text { metabólica }\end{array}$ & $\begin{array}{c}\text { Abril a } \\
\text { novembro de } \\
2015 \\
\text { (8 meses) } \\
\text { Encontros } \\
\text { mensais, com } \\
\text { duração entre } \\
90 \text { min e } 120 \\
\text { min cada. }\end{array}$ & $\begin{array}{l}\text { Os participantes foram } \\
\text { expostos a oficinas e } \\
\text { dinâmicas, com atividades } \\
\text { interativas, rodas de } \\
\text { conversa e distribuição } \\
\text { de folhetos explicativos } \\
\text { sobre o tema abordado. }\end{array}$ & $\begin{array}{l}\text { Após cada encontro } \\
\text { eram aferidos } \\
\text { peso corporal e } \\
\text { circunferência da } \\
\text { cintura. No último } \\
\text { encontro, mediante } \\
\text { uma dinâmica, } \\
\text { os participantes } \\
\text { foram questionados } \\
\text { sobre hábitos } \\
\text { que conseguiram } \\
\text { modificar após o } \\
\text { processo. }\end{array}$ & $\begin{array}{c}\text { Participantes } \\
\text { consolidaram seus } \\
\text { conhecimentos e } \\
\text { a autonomia para } \\
\text { escolhas alimentares } \\
\text { saudáveis e } \\
\text { sentiram-se mais } \\
\text { seguros e motivados } \\
\text { em superar as } \\
\text { dificuldades durante } \\
\text { o tratamento } \\
\text { nutricional. }\end{array}$ \\
\hline $\begin{array}{l}\text { De Melo et } \\
\text { al., } 2016\end{array}$ & $\begin{array}{c}\text { Relatar uma } \\
\text { experiência } \\
\text { de estágio de } \\
\text { nutrição no NASF em } \\
\text { Unidades de Saúde } \\
\text { da Família }\end{array}$ & $\begin{array}{l}\text { Usuários de duas } \\
\text { Unidades de } \\
\text { Saúde da Família. } \\
\text { Palmas-TO }\end{array}$ & $\begin{array}{l}\text { Maio a junho } \\
\text { de } 2015 . \\
\text { (2 meses) }\end{array}$ & $\begin{array}{l}\text { Foram realizadas } \\
\text { apresentações } \\
\text { participativas sobre } \\
\text { alimentação saudável, } \\
\text { rodas de conversa e } \\
\text { atividades coletivas } \\
\text { com grupos variados de } \\
\text { usuários da Unidade }\end{array}$ & $\begin{array}{c}\text { Em algumas } \\
\text { atividades foi } \\
\text { realizado um } \\
\text { questionamento } \\
\text { informal aos } \\
\text { participantes, mas } \\
\text { não houve avaliação } \\
\text { inicial e final em todas } \\
\text { as atividades. }\end{array}$ & $\begin{array}{l}\text { Não foi possível } \\
\text { identificar resultados } \\
\text { das intervenções. }\end{array}$ \\
\hline $\begin{array}{l}\text { Deus et al., } \\
2015\end{array}$ & $\begin{array}{l}\text { Avaliar o impacto } \\
\text { de intervenção } \\
\text { nutricional, em } \\
\text { conjunto com } \\
\text { prática de exercício } \\
\text { físico, sobre o } \\
\text { perfil alimentar e } \\
\text { antropométrico de } \\
\text { usuárias do Programa } \\
\text { Academia da Saúde. }\end{array}$ & $\begin{array}{l}124 \text { mulheres } \\
\text { participantes } \\
\text { do Programa } \\
\text { Academia da } \\
\text { Saúde de Belo } \\
\text { Horizonte, MG. }\end{array}$ & $\begin{array}{l}11 \text { meses } \\
\text { Encontros } \\
\text { mensais, com } \\
\text { duração de } \\
60 \text { min cada. }\end{array}$ & $\begin{array}{l}\text { A intervenção nutricional } \\
\text { foi realizada com o uso } \\
\text { de ilustrações por meio } \\
\text { de materiais educativos } \\
\text { e lúdicos, como réplicas, } \\
\text { fotos de alimentos e } \\
\text { medidas caseiras, além de } \\
\text { jogos educativos e teatro, } \\
\text { bem como de atividades } \\
\text { pelo educador físico. }\end{array}$ & $\begin{array}{c}\text { Para analisar o } \\
\text { impacto da avaliação } \\
\text { foi considerada } \\
\text { a evolução de } \\
\text { indicadores dietéticos } \\
\text { e antropométricos no } \\
\text { início da intervenção } \\
\text { e após o período de } \\
11 \text { meses. }\end{array}$ & $\begin{array}{l}\text { Impacto positivo } \\
\text { nos indicadores } \\
\text { nutricionais, } \\
\text { antropométricos e } \\
\text { bioquímicos. }\end{array}$ \\
\hline
\end{tabular}




\begin{tabular}{|c|c|c|c|c|}
\hline $\begin{array}{l}\text { Frois, } \\
\text { Dourado e } \\
\text { Pinho, } 2016 .\end{array}$ & $\begin{array}{l}\text { Relatar a experiência } \\
\text { da implantação de } \\
\text { ações educativas } \\
\text { sobre alimentação } \\
\text { saudável entre } \\
\text { profissionais de saúde } \\
\text { na Estratégia Saúde } \\
\text { da Família. }\end{array}$ & $\begin{array}{c}50 \text { equipes } \\
\text { de Estratégia } \\
\text { Saúde da Família } \\
\text { integrantes } \\
\text { do projeto } \\
\text { das Unidades } \\
\text { Promotoras de } \\
\text { Saúde, na cidade } \\
\text { de Montes Claros } \\
\text { - MG. }\end{array}$ & $\begin{array}{c}\text { Dezembro de } \\
2014 \text { a março } \\
\text { de } 2015 \\
\text { (4 meses) } \\
4 \text { encontros } \\
\text { com duração } \\
\text { de } 50 \text { min } \\
\text { cada. }\end{array}$ & $\begin{array}{l}\text { Os participantes } \\
\text { foram expostos a } \\
\text { oficinas e dinâmicas } \\
\text { que proporcionam } \\
\text { participação ativa dos } \\
\text { indivíduos. }\end{array}$ \\
\hline
\end{tabular}

\begin{tabular}{lc}
\hline Machado et & Comparar o efeito de \\
al., 2016 & três estratégias de \\
& educação em saúde \\
& e nutrição sobre a \\
& adesão ao tratamento \\
& não farmacológico da \\
& Hipertensão Arterial \\
& Sistêmica (HAS).
\end{tabular}

\section{2 indivíduos} com diagnóstico médico de HAS, acompanhados pelo Programa Hiperdia.

Divididos em três grupos de intervenção, sendo:

G1 (de 8 a 12 oficinas)

G2 (oficina + VD)

G3 (menos de 8 oficinas).

\begin{tabular}{lc}
\hline Gomes et al., & Avaliar o impacto \\
de um programa & de educação \\
& nutricional sobre o \\
estado nutricional & e conhecimento \\
alimentar de \\
pacientes com \\
excesso de peso.
\end{tabular}

\section{Pereira et al., Relatar a experiência} 2015 das atividades de Educação Alimentar e Nutricional (EAN) desenvolvidas em pacientes com sobrepeso e obesidade.
15 pacientes

frequentadores de um grupo de educação alimentar para pessoas com excesso de peso.

$$
\begin{aligned}
& \text { Julho de } \\
& 2012 \text { a }
\end{aligned}
$$

setembro de

2013

(14 meses)

1 oficina por mês com duração

aprox. 1 hora e visitas domiciliares de $45 \mathrm{~min}$.
Foram realizadas oficinas, palestras dialogadas e dinâmicas interativas com a utilização de recursos diversos, como cartazes, vídeos e demonstrações práticas. Nas visitas domiciliares eram realizadas orientações práticas de acordo com a realidade de cada família.
Para avaliar o aprendizado adquirido foram aplicados questionários individuais sobre todas as atividades realizadas.
Esta ação proporcionou a construção de novos conceitos sobre alimentação saudável entre os profissionais de saúde.

\section{Avaliação dietética, variáveis antropométricas, bioquímicas, pressão arterial, atividade física e informações sobre o consumo de alimentos, foram coletadas antes e depois das intervenções.}

As três intervenções educativas obtiveram resultados positivos. Os resultados indicaram melhora nos parâmetros antropométrico, bioquímicos e dietéticos em todos os grupos.

Setembro a Desenvolveram a $\quad$ Medidas

outubro de

( 2 meses)

7 encontros semanais.

educação nutricional por meio de aulas expositivas e conversas em grupo, discutindo diferentes temas referentes à alimentação saudável e aplicação de questionários para avaliar o nível de conhecimento dos pacientes.

\section{6 pacientes \\ Setembro \\ Os temas foram} com sobrepeso e obesidade graus I e II atendidos no ambulatório do Hospital Universitário da Universidade Federal de Juiz de Fora integrantes do projeto de extensão "Saúde na Balança". de 2012 a

setembro de

2013

(12 meses)

Sendo os encontros: Semanais no 10 mês, quinzenais no 2 e

3 으ês e mensais 4 o ao 60 mês. abordados nos encontros utilizando recursos materiais tais como réplicas e rótulos de alimentos, fotos, jogos de tabuleiro, competição, cruzadinha, memória e elaboração de receitas saudáveis. antropométricas

foram realizadas no início e no final da intervenção, bem como aplicação de um questionário para avaliar o nível de conhecimento antes e após cada encontro.

\section{Ao final de cada} encontro os participantes recebiam uma ficha de avaliação para que pudessem dar a sua opinião sobre o conteúdo e estratégias utilizadas.
Redução das medidas antropométricas, com redução de peso, evolução no estado nutricional e melhoria no conhecimento sobre alimentação saudável.

Os resultados foram positivos no que se refere à melhor compreensão das orientações nutricionais dadas nas consultas individuais dos participantes.

\begin{tabular}{lcc}
\hline $\begin{array}{l}\text { Rosa, Giusti } \\
\text { e Ramos, }\end{array}$ & $\begin{array}{c}\text { Identificar e ampliar } \\
\text { os conhecimentos }\end{array}$ & $\begin{array}{c}8 \text { universitários } \\
\text { de diferentes } \\
\text { 2016. }\end{array}$ \\
$\begin{array}{ll}\text { sobre alimentos } \\
\text { para contribuir na } \\
\text { mudança de hábitos }\end{array}$ & $\begin{array}{c}\text { residentes da } \\
\text { Casa do Estudante }\end{array}$ \\
& e práticas alimentares & em Porto Alegre \\
inadequadas de & RS \\
adultos jovens & \\
residentes em & \\
moradia estudantil & \\
em Porto Alegre RS.
\end{tabular}

$\begin{array}{cc}\text { Foram realizados } & \text { Ao final do processo } \\ \text { encontros com o uso } & \text { de intervenção } \\ \text { de materiais didáticos e } & \text { foram aplicados } \\ \text { conversas participativas } & \text { questionários do } \\ \text { cobre Educação Alimentar } & \text { comportamento } \\ \text { e Nutricional. } & \text { alimentar, } \\ & \text { autoavaliação } \\ & \text { e avaliação do } \\ & \text { projeto para avaliar } \\ & \text { efetividade. }\end{array}$

Foi possível identificar e ampliar os conhecimentos sobre alimentos/ alimentação, o que contribuiu para a mudança de alguns hábitos e práticas inadequadas dos participantes. 
Observou-se diversidade nos objetivos das intervenções de EAN. Três estudos (BRITO et al., 2017; DO NASCIMENTO et al., 2016; PEREIRA et al., 2015) tiveram como objetivo relatar a experiência de aplicação de práticas de EAN com grupos específicos. Frois, Dourado e Pinho (2016) tinham como proposta relatar a experiência da instituição de ações educativas sobre alimentação saudável destinadas a profissionais de saúde, enquanto De Melo et al. (2016) relataram a experiência com EAN, do estágio de nutrição, nos Núcleos de Apoio à Saúde da Família. Outros três estudos (BELEDELLI; SANTOLIN, 2017; DEUS et al., 2015; GOMES et al., 2013) tinham como objetivo avaliar o impacto de intervenções de EAN com o grupo estudado: pacientes com excesso de peso; usuários do Programa Academia da Saúde e praticantes de pilates. Cabe referir que esses três estudos tinham como proposta aliar a intervenção de EAN com a prática de exercícios físicos. Já Rosa, Giusti e Ramos (2016) buscaram identificar e ampliar os conhecimentos sobre alimentos a fim de contribuir para a mudança de hábitos e de práticas alimentares inadequadas no grupo estudado e Machado et al. (2016) compararam e analisaram três estratégias de educação em saúde para o grupo em estudo.

Ao analisar o público-alvo das intervenções, constatou-se que os autores trabaIharam a EAN com grupos diversos. Parte dos estudos estava centrada em intervenções destinadas a pacientes portadores de alguma morbidade: síndrome metabólica (Brito et al., 2017), doença renal crônica (DO NASCIMENTO et al., 2016), hipertensão (MACHADO et al., 2016), excesso de peso (PEREIRA et al., 2015; GOMES et al., 2013). Outro grupo de estudos teve participantes que, necessariamente, não apresentavam alguma doença: profissionais de saúde (FROIS; DOURADO; PINHO 2016); usuários de Unidade de Saúde (DE MELO et al., 2016; DEUS et al., 2015); praticantes de pilates, usuários de academia (BELEDELLI; SANTOLIN, 2017); e universitários residentes em moradia estudantil (ROSA; GIUSTI; RAMOS, 2016).

Quanto ao desenvolvimento da ação educativa, constatou-se que o período de duração variou de 2 a 14 meses, destacando-se que três estudos tiveram duração maior que 10 meses. Quando informado pelos autores, a maioria das intervenções ocorreu com frequência mensal. No estudo de Gomes et al. (2013) a frequência dos encontros foi semanal, e no de Pereira et al. (2015) as atividades do grupo ocorreram semanalmente no 1 으 mês, quinzenalmente no 20 e 30 mês e mensalmente até o 60 mês.

Em relação à metodologia utilizada nas ações de EAN, identificou-se que os autores apresentaram diferentes formas de conduzir suas intervenções educativas. Observa-se que, na grande maioria dos estudos, as ações de EAN foram realizadas no modelo de oficinas participativas, utilizando rodas de conversa e dinâmicas em grupo, por exemplo, atividades de "verdadeiro ou falso" ou "mitos e verdades" sobre alimentação saudável (DO NASCIMENTO et al., 2016; ROSA; GIUSTI; RAMOS, 2016), teatro (DEUS et al., 2015), e leitura e interpretação de rótulos de produtos alimentícios (GOMES et al., 2013; DO NASCIMENTO et al., 2016; FROIS; DOURADO; PINHO, 2016; ROSA; GIUSTI; RAMOS, 2016). Além disso, os autores realizaram diversas atividades lúdicas - cruzadinhas, jogos educativos, desenho da refeição habitual no prato - e também utilizaram diferentes materiais e recursos: réplicas de alimentos e fotos e/ou medidas caseiras dos alimentos, por exemplo. 
Constata-se também que foram utilizados recursos audiovisuais - slides e projeção multimídia nesses estudos. Gomes et al. (2013) conduziram os encontros mediante aulas expositivas e conversas, e Brito et al. (2017) reproduziram slides com ilustrações e explicações sobre o tema de cada oficina, associados à distribuição de folhetos explicativos. Também foram utilizados sessão de cinema, cartazes informativos, lanches coletivos e a elaboração de receitas saudáveis, em que cada participante preparou uma receita, com base nos aprendizados ao longo do processo, para confraternização ao final do último encontro.

Identificou-se a preocupação de alguns autores em relação às atividades educativas, a fim de que estimulassem o diálogo e a problematização, possibilitando a troca de vivências e de experiências (MACHADO et al., 2016), proporcionassem a construção de conceitos aplicáveis à realidade dos participantes (DEUS et al., 2015), fundamentando-se na teoria da dialogicidade de Paulo Freire (ROSA;GIUSTI; RAMOS, 2016). Cabe referir que Beledelli e Santolin (2017) não citaram a metodologia utilizada nas intervenções educativas.

As avaliações das intervenções ocorreram no pré e pós-intervenção, ou seja, foi avaliado o perfil antropométrico, perfil dietético (por meio de questionários de hábitos alimentares) e perfil bioquímico, antes e após o período estudado, e Gomes et al. (2013) e Machado et al. (2016) realizaram aferição da pressão arterial além dos demais indicadores. Dois autores (PEREIRA et al., 2015; GOMES et al., 2013) aplicaram um questionário ao final de cada encontro para analisar a efetividade da intervenção. Pereira et al. (2015) entregaram uma ficha de avaliação ao final de cada encontro para que os participantes pudessem avaliar o conteúdo e as estratégias de educação utilizadas, e solicitaram sugestões para melhoria do projeto. Brito et al. (2017) realizaram aferição de peso e circunferência da cintura ao final de cada encontro para o acompanhamento da evolução dos participantes.

Quanto aos resultados, com exceção de um estudo (DE MELO et al., 2016), todos os outros analisados referiram resultados positivos após a intervenção, com o impacto positivo nos indicadores nutricionais, antropométricos e bioquímicos. Além disso, relataram melhoria na adesão ao tratamento de indivíduos diagnosticados com sobrepeso, obesidade, hipertensão, doença renal crônica e síndrome metabólica. Pôde-se também observar um impacto positivo no conhecimento e na autonomia para escolhas alimentares mais saudáveis. Brito et al. (2017), por exemplo, destacam que os participantes consolidaram seus conhecimentos sobre alimentação, puderam desenvolver a autonomia para escolhas alimentares saudáveis e sentiram-se mais motivados a superar as dificuldades durante o tratamento nutricional.

$\mathrm{Na}$ análise das referências bibliográficas dos artigos, identificou-se que em nenhum dos estudos os autores citaram a utilização do Marco de EAN. Diante desse contexto, optou-se por verificar se, indiretamente, o conteúdo do documento estava presente nos artigos. Em relação à presença dos princípios do Marco de EAN, constatou-se que, praticamente, todos os artigos apresentaram alguma abordagem relacionada a um ou mais dos princípios do Marco, mesmo que nenhum deles tenha utilizado diretamente ou explicitamente o Marco de EAN, seja como referência na descrição metodológica ou como base teórica na construção do texto. Sendo assim, a partir da análise aqui rea- 
lizada, conforme consta no Quadro 1, foi possível identificar algumas possibilidades de aproximação dos princípios propostos no Marco de EAN e os artigos selecionados para análise.

O princípio que, mesmo não fazendo alusão direta ao Marco, esteve presente em mais estudos foi o Princípio 6 - a educação como processo permanente e gerador de autonomia e participação ativa, aparecendo em seis ações (BRITO et al., 2017; DE MELO et al., 2016; FROIS; DOURADO; PINHO, 2016; MACHADO et al., 2016; GOMES et al., 2013; PEREIRA et al., 2015). Em seguida, apareceu o Princípio 9 - planejamento, avaliação e monitoramento das ações, em quatro estudos (BRITO et al., 2017; DEUS et al., 2015; PEREIRA et al., 2015; ROSA; GIUSTI; RAMOS, 2016).

Chamou a atenção o fato de que os temas relacionados aos Princípios 1, 2, 5 e 8 do Marco, mesmo que indiretamente, não foram abordados por nenhum dos autores.

Quadro 1 - Identificação dos estudos que abordaram o Marco e resumos dos princípios utilizados

Autor

Princípio(s) do Marco utilizados

\begin{tabular}{|c|c|}
\hline Beledelli; Santolin, 2017 & $\begin{array}{l}\text { Não foi identificada a aproximação com os princípios do Marco de } \\
\text { EAN no estudo desses autores. }\end{array}$ \\
\hline \multirow[t]{5}{*}{ Brito et al., 2017} & $\begin{array}{l}\text { Princípio 3: Durante as oficinas de EAN foi solicitado aos } \\
\text { participantes que trouxessem receitas de preparações caseiras, } \\
\text { valorizando, desse modo, especificidades culturais de cada } \\
\text { participante. }\end{array}$ \\
\hline & $\begin{array}{l}\text { Princípio 4: Os participantes foram estimulados a trazer } \\
\text { receitas de preparações caseiras que consideravam saudáveis, } \\
\text { estimulando a culinária como recurso para alimentação saudável. }\end{array}$ \\
\hline & $\begin{array}{l}\text { Princípio 6: Os participantes foram estimulados a refletir } \\
\text { sobre os principais fatores limitantes na adesão às orientações } \\
\text { nutricionais. }\end{array}$ \\
\hline & $\begin{array}{l}\text { Princípio 7: Os temas de encontro eram conduzidos de } \\
\text { forma coordenada e as abordagens eram complementares às } \\
\text { particularidades do grupo em estudo. }\end{array}$ \\
\hline & $\begin{array}{l}\text { Princípio 9: Os temas das ações de EAN foram planejados em } \\
\text { conjunto, e foram estabelecidas metas para serem alcançadas ao } \\
\text { final da intervenção, quando ocorreu a avaliação do processo. }\end{array}$ \\
\hline De Melo et al., 2016 & $\begin{array}{l}\text { Princípio 6: Nas atividades realizadas, os participantes foram } \\
\text { incentivados a participar de maneira ativa com questionamentos } \\
\text { e reflexões sobre o tema abordado. }\end{array}$ \\
\hline Deus et al., 2015 & $\begin{array}{l}\text { Princípio 9: Os temas das ações de EAN foram planejados em } \\
\text { conjunto, respeitando o perfil dos participantes, o objetivo da } \\
\text { intervenção era claro e ao final da intervenção ocorreu uma } \\
\text { avaliação do processo. }\end{array}$ \\
\hline Do Nascimento et al., 2016 & $\begin{array}{l}\text { Princípio 7: Os temas dos encontros realizados eram adequados } \\
\text { ao cenário e às especificidades do grupo de participantes. }\end{array}$ \\
\hline $\begin{array}{l}\text { Frois, Dourado e Pinho, } \\
2016\end{array}$ & $\begin{array}{l}\text { Princípio 6: Durante as oficinas foi criado um espaço que } \\
\text { propiciava a socialização e troca de experiências entre os } \\
\text { participantes; estimulou-se o pensamento crítico na construção } \\
\text { do conhecimento para garantir o empoderamento do sujeito. }\end{array}$ \\
\hline
\end{tabular}




\section{Machado et al., $2016 \quad$ Princípio 6: A troca de vivências e experiências entre os} participantes foi estimulada, o modelo de educação em saúde utilizado foi baseado na interatividade e no diálogo.

Princípio 7: A temática da intervenção levou em consideração a realidade da população atendida.

Gomes et al., 2013 Princípio 6: Os integrantes do grupo foram estimulados a relatar os temas de interesse e foram trabalhados aspectos emocionais dos participantes, com o intuito de que a convivência e apresentação de vivências facilitassem o aprendizado.

Pereira et al., 2015 Princípio 6: Os temas abordados durante os encontros buscaram a participação efetiva dos participantes.

Princípio 9: Os autores realizaram um diagnóstico para identificar quais aspectos relacionados à alimentação e à saúde eram de interesse dos participantes.

Rosa, Giusti e Ramos, 2016 Princípio 9: Identificou-se que os autores realizaram um diagnóstico sobre temas de interesse dos participantes; foi aplicado um questionário para identificar o comportamento alimentar do grupo. Com esses dados levantados no diagnóstico foi realizado um planejamento para que a intervenção abordasse os temas sugeridos pelos participantes.

Fonte: Elaboração própria.

Quanto ao uso de abordagens educacionais ativas e problematizadoras, identificou-se que Brito et al. (2017) preocuparam-se em estimular os participantes a refletir sobre as questões problematizadoras e as limitações que impedem uma alimentação saudável, e, com esses dados identificados, trabalharam a EAN sob as perspectivas dos integrantes do grupo. Também se identificou que Machado et al. (2016) trabalharam a EAN com um modelo de educação em saúde baseado na interatividade, no diálogo e na problematização da realidade dos indivíduos. No estudo de Rosa, Giusti e Ramos (2016) a intervenção baseou-se na teoria da dialogicidade descrita por Paulo Freire, considerando as características dos indivíduos, de maneira que a reflexão e o diálogo estivessem presentes em todos os encontros.

Frois, Dourado e Pinho (2016), que realizaram o estudo com profissionais de saúde na Estratégia Saúde da Família, ressaltaram a importância de considerar o meio em que o indivíduo está inserido e utilizar estratégias para promover uma aprendizagem efetiva, embasada no pensamento reflexivo, dialógico, contextual, colaborativo e construtivo. Durante os encontros os autores estimularam, nos participantes, um pensamento crítico na construção do conhecimento.

\section{DISCUSSÃO}

A seleção de artigos com a temática da EAN com adultos, no período de 2012 a 2018, partiu da necessidade de se identificar e configurar as intervenções realizadas após a criação do Marco de Referência de Educação Alimentar e Nutricional para Políticas Públicas. Nesse sentido, considerando-se o reduzido número de publicações encontradas que abordavam o conceito "Educação Alimentar e Nutricional", no período analisado, identificou-se a necessidade de que este tema, além de ser mais amplamente discutido, também tenha como fundamentação esse importante produto fruto do debate amplo entre especialistas da área, que é o Marco de Referência. 
Quanto à análise dos objetivos das ações, constatando-se que cinco estudos tinham por propósito a intervenção, com base na adesão ao tratamento de doenças, percebe-se que as ações de EAN são fundamentais, não somente na prevenção e promoção da saúde, mas também no tratamento e melhora nos níveis bioquímicos, antropométricos e dietéticos dos sujeitos.

Cervato-Mancuso (2013) confirma que a seleção adequada dos objetivos é o ponto fundamental para o êxito do programa de EAN, o que exige um conhecimento do público-sujeito - seus hábitos alimentares e todo o contexto em que vive - realizado por meio do diagnóstico educativo. Corroborando essa constatação, Esperança e Galisa (2014) afirmam que é necessário ter claramente definido o que se espera atingir com a ação, ou seja, o objetivo, antes de iniciar o processo de EAN.

A partir dos resultados encontrados nos artigos aqui pesquisados, porém, também é possível afirmar que são necessários mais ações e estudos que abordem a EAN para públicos sem patologias associadas e com o intuito de se trabalhar a promoção de saúde contextualizada na realidade dos indivíduos (FRANÇA; CARVALHO, 2017). Boog (2013) reforça que muitos estudos na área da EAN apresentam ações educativas descontextualizadas da realidade da população-alvo, evidenciando, possivelmente, a falta da etapa de planejamento e também de ações pontuais e de curta duração, as quais, dificilmente, seriam capazes de atingir os objetivos propostos.

Quanto ao público-alvo dos estudos, observou-se uma faixa etária variada, com indivíduos com idade de 18 anos ou mais, e somente em dois dos estudos havia idosos entre os participantes. As intervenções podem e devem ser específicas para as diferentes faixas etárias, pois sabe-se que isso proporcionará maior compreensão por parte dos indivíduos (FRANÇA; CARVALHO, 2017). O que chama a atenção, no entanto, é que em nenhum dos estudos o foco principal eram os sujeitos e sim a patologia associada, o perfil nutricional, o conhecimento ou o relato da experiência. Outro elemento observado é que o sexo feminino teve destaque entre os participantes. Gomes et al. (2013) justificam a participação maior de mulheres pelo fato de serem as principais usuárias de serviços de saúde, em diferentes fases da vida, e por terem uma preocupação maior com a saúde quando comparadas aos homens.

As ações/intervenções realizadas não foram descritas em detalhes em boa parte dos estudos, dificultando o seu entendimento e a análise, o que corrobora o estudo de Santos (2012), quando este observa que os estudos em EAN não costumam descrever, de forma aprofundada, as atividades desenvolvidas no processo educativo. Nesse sentido, o que se pode assimilar da metodologia utilizada nos artigos analisados é que, nas ações, ainda se destacam os artifícios como palestras e métodos expositivos, o que, segundo Santos (2012), pode favorecer a apresentação do tema exposto ao grupo, porém essas ações acabam caindo em tipos de técnicas associadas a um modelo de educação tradicional, de transmissão de conhecimentos e verticalidade na relação entre educador e educando.

Nesse sentido, cabe mencionar a importância do planejamento da intervenção educativa, tendo bem claro os objetivos que se pretende com a ação e a metodologia que será utilizada, pois a EAN deve ampliar sua abordagem para além da transmissão de conhecimento, gerando situações de reflexão sobre as práticas alimentares. Santos 
(2012), ao analisar as práticas educativas de EAN, observou um distanciamento entre o referencial teórico apresentado como base para o planejamento e as ações efetivamente desenvolvidas na prática.

Sobre a avaliação e os resultados da intervenção, os indicadores que avaliam modificações biológicas e corporais são os mais utilizados, destacando modificações/resultados positivos. $\mathrm{O}$ uso desses indicadores justifica-se pelo fato de que muitas das intervenções centraram-se em grupos de pessoas com doenças que se pretendia intervir. Questiona-se, todavia, se esses resultados seriam duradouros, considerando-se que não sinalizavam avaliações posteriores ao período das intervenções.

Boog (2013, p. 254) também refere que, em boa parte dos estudos em Educação Alimentar e Nutricional, os resultados apresentam apenas a mensuração de conhecimentos sobre nutrição ou a mudança de práticas alimentares. Para essa autora, as avaliações que se preocupam em mostrar resultados numéricos "não refletem realmente o significado daquela ação educativa na vida das pessoas". A autora critica o uso desses dados, apontando a necessidade de avaliar resultados não mensuráveis, pois a alimentação é complexa e envolve fatores muito além do alimento, entre os quais os sentimentos e valores.

França e Carvalho (2017) afirmam que a simples avaliação do conhecimento adquirido por si só não leva à confirmação de mudança de comportamento alimentar dos sujeitos, fazendo-se necessária uma avaliação mais crítica da intervenção, partindo da complexidade dos sujeitos, não se apoiando apenas em resultados objetivos. Por isso, recomendam que a avaliação seja um processo contínuo, a fim de verificar se os objetivos propostos no planejamento foram alcançados, e identificar adequações, reformulações ou novas necessidades para futuras ações (ESPERANÇA; GALISA, 2014; CERVATO-MANCUSO, 2013).

Quanto aos princípios do Marco de EAN, o uso do princípio 6, em seis dos estudos aqui analisados, mesmo que de forma indireta, sugere que, com essas ações realizadas, os autores tiveram a preocupação de fortalecer a participação ativa dos indivíduos, como se identificou em Frois, Dourado e Pinho (2016), em cujo estudo os autores criaram um espaço que propiciava a socialização e troca de experiências entre os participantes, estimulando o pensamento crítico na construção do conhecimento para garantir o empoderamento do sujeito.

Ao se identificar que somente quatro dos estudos analisados realizaram as ações com o uso de abordagens e recursos educacionais problematizadores e ativos que favorecessem o diálogo entre os participantes, destaca-se que essa abordagem ainda é incipiente. Isto porque demonstra uma fragilidade metodológica e de planejamento das ações, pois, como é preconizado por Paulo Freire, o diálogo é fundamental para fazer com que os participantes possam refletir sobre sua história e realidade e assim buscar soluções (FREIRE, 2004). Esperança e Galisa (2014) consideram importante que o educador trabalhe com atividades em que os participantes sejam estimulados a refletir sobre os benefícios da adoção voluntária de práticas alimentares saudáveis. Considerando-se o atual papel da EAN, destaca-se a necessidade de favorecer o diálogo nas ações propostas, porque este oferece sentido às ações educativas e ao processo de mudança na alimentação das populações (SCARPARO, 2017). 
De modo geral, nos estudos avaliados, observa-se certa despreocupação com o processo da ação educativa como um todo, ou seja, desde o planejamento da ação até a avaliação e monitoramento. O Princípio 9, que trata desse tema, mesmo sendo o segundo mais utilizado, é abordado em apenas quatro estudos. Esse princípio reforça que também seria necessário que todos os implicados na ação (profissionais e público-alvo) estivessem envolvidos com o processo, pois as ações com metas estabelecidas de forma participativa tendem a ter melhores resultados, impacto e sustentabilidade nas iniciativas (BRASIL, 2012b).

Para se executar um planejamento de intervenções educativas, com o intuito de realizar uma intervenção de EAN, é necessário, inicialmente, haver um diagnóstico, ou seja, uma demanda. O segundo ponto do planejamento é o que se pretende mudar com essa intervenção, e o terceiro é a definição do conteúdo abordado para alcançar os objetivos, a elaboração da metodologia e as estratégias. Por fim, a última etapa é a realização de tudo o que foi planejado (FAGIOLI; NASSER, 2006). Durante a elaboração do programa de EAN, alguns determinantes devem ser considerados, quais sejam: fatores individuais, sociais, econômicos e culturais. Esses determinantes influenciam diretamente as práticas alimentares (BRASIL, 2014b). Nota-se que esse pensamento vem crescendo, porém ainda são necessárias mais publicações na área para que isso seja concretizado, além do fornecimento de ações de formação para os atores envolvidos com o planejamento e desenvolvimento das ações de EAN.

Em apenas um dos estudos foi identificado o Princípio 3, que destaca a importância de se inserir a cultura alimentar local nas ações de EAN, valorizando a diversidade étnica e cultural do Brasil. A alimentação faz parte da identidade e da cultura de uma população, e discutir essa temática em uma ação de EAN enriquece os saberes e proporciona seu compartilhamento (BRASIL, 2012b). A Política Nacional de Alimentação e Nutrição (PNAN) chama a atenção para a importância do respeito à diversidade e à cultura alimentar, pois a alimentação brasileira, com suas particularidades regionais, é a síntese do processo histórico de intercâmbio cultural (BRASIL, 2012a). Há uma diversidade sociocultural no Brasil - reflexo de influências de práticas e saberes alimentares dos diferentes povos que constituíram nossa população - que precisam ser respeitadas nas ações de EAN.

Na presente análise também identificou-se que apenas um dos estudos abordou o Princípio 4, que contém a temática da comida e do alimento como referência no lugar dos nutrientes e a valorização da culinária. Esse princípio vai ao encontro do que preconiza o Guia Alimentar para a População, ao orientar as pessoas a optarem por alimentos in natura, refeições caseiras no lugar de produtos prontos e ultraprocessados. Também há uma atenção especial às circunstâncias que envolvem o ato de comer, aconselhando-se a regularidade de horário, ambientes apropriados e, sempre que possível, companhia (BRASIL, 2014c).

Sobre a abordagem do Princípio 1, chama a atenção o fato de que nenhum dos estudos discutiu o tema sustentabilidade, refletindo uma deficiência no planejamento das ações, pois este é um tema atual e há necessidade de ser utilizado em diversos setores. Ribeiro, Jaime e Ventura (2017) sinalizam as inúmeras interfaces existentes na relação entre a alimentação (considerando o amplo e complexo trajeto do alimento da produ- 
ção até o consumo) e a sustentabilidade, chamando a atenção para a visão sistêmica da alimentação no conceito de alimentação saudável, que implica reflexão sobre todo o sistema alimentar. Nesse sentido, em relação ao Princípio 2, o qual sugere abordagens sobre o sistema alimentar na sua integridade, não foram constatadas discussões em nenhum dos estudos analisados. Isso revela que ações em torno da alimentação saudável, em uma concepção mais ampla e que poderiam orientar novas práticas e escolhas individuais mais conscientes, ainda são escassas.

O Princípio 5, igualmente não abordado em nenhum dos estudos, sugere que o cuidado de si, considerando a integralidade das dimensões corporais (física, mental e espiritual) não é um tema usual em ações. O cuidado de si, quando se realiza, passa a ser existencial e a ser sentido e vivido, refletindo-se no ambiente, pois ele revela nossa forma de atuar, nossa atitude em relação ao mundo, comunicando nossa forma de ser (SILVA, 2010). Assim, é necessária uma revisão das abordagens usuais no contexto de EAN, no sentido de promover o autocuidado dos indivíduos.

Constatou-se que, em seis dos dez estudos analisados, a equipe que realizou a ação de EAN era composta somente por nutricionista e estudantes de Nutrição. Esse dado faz refletir sobre o Princípio 8, não presente na totalidade dos estudos, que sugere que as ações precisam ser realizadas de forma além de interprofissional, também intersetorial, o que possibilita ações mais eficientes e que produzam soluções inovadoras (BRASIL, 2012b). A Política Nacional de Promoção de Saúde (PNPS) reforça que a promoção da saúde, como prática social intersetorial, está diretamente relacionada à qualidade de vida e ao equilíbrio do processo saúde-doença. Esse documento destaca que a promoção da saúde legitima a articulação sujeito/coletivo, público/privado, estado/ sociedade, clínica/política, setor sanitário/outros setores, proporcionando o rompimento com a fragmentação da abordagem do processo saúde-doença, suprimindo vulnerabilidade, riscos e danos que nela se produzam (BRASIL, 2006).

Por fim, com base no exposto, constata-se que há necessidade de mais ações e estudos que foquem o Marco de Referência em EAN e as práticas problematizadoras. Cervato-Mancuso et al. (2016) sugerem que pensar em intervenção grupal é pensar em prática social, o que exigiria, assim, um profissional mais bem preparado e hábil para a realização da ação, e também a utilização de referências reconhecidas, entre as quais o Marco de EAN.

\section{CONSIDERAÇÕES FINAIS}

As primeiras políticas públicas e intervenções na área da alimentação e da nutrição, em nosso país, centravam-se em distribuir alimentos e garantir que a população tivesse acesso a eles. Com a inversão no padrão alimentar da população brasileira, a EAN voltou-se para a promoção da saúde e para a prevenção de doenças relacionadas à má alimentação.

Em 2012 foi lançado o Marco de Referência de Educação Nutricional e Alimentar para Políticas Públicas, com o intuito de ser um documento de orientação prática para planejamento de ações de EAN. Assim, no presente estudo, buscou-se traçar um panorama das intervenções em educação alimentar e nutricional com adultos, após a criação do Marco de Referência. 
Dos 11 estudos aqui analisados, 10 deles abordaram, no mínimo, um dos princípios do Marco de EAN, mas nenhum deles o utilizou diretamente como referência na descrição metodológica ou como base teórica para a elaboração do texto. Constatou-se, então, que, para o planejamento das intervenções, não houve a valorização desse documento como referência da área. Além disso, também não foram abordados temas emergentes na ciência da Nutrição, entre os quais: sustentabilidade social, ambiental e econômica; sistema alimentar na sua integralidade; cultura alimentar e valorização da culinária.

Os estudos analisados permitem traçar um mapa inicial a respeito de ações de EAN com adultos a partir do marco de referência, porém cabe ressaltar que são em pequeno número, além de serem muito distintos entre si, seja em relação ao público ou às metodologias adotadas.

As intervenções são realizadas em curto intervalo de tempo, caracterizando-se por serem ações pontuais, não inseridas em um programa maior de EAN, o que, se sabe, tende a tornar seus resultados limitados no médio e no longo prazo. Isso pode estar relacionado aos objetivos que se quer da EAN. A partir de perspectivas educacionais tradicionais, existe a crença de que a transmissão da informação seria o suficiente para a aprendizagem e posterior mudança de atitudes. Nesse contexto, permanece a dúvida sobre os seus reais resultados, apesar da apresentação de bons indicativos.

Evidencia-se que, embora haja uma preocupação crescente na literatura em relação às bases teórico-metodológicas que regem as ações educativas em alimentação e nutrição, os estudos analisados, em grande parte, inseriram-se em modelos tradicionais, não abordando questões educacionais problematizadoras que façam o indivíduo refletir e agir em relação à alimentação.

Finalmente, destaca-se a necessidade de construção de novas perspectivas para as práticas de educação alimentar e nutricional, e a necessidade de aperfeiçoar os estudos mediante a utilização do Marco de EAN como referência.

\section{REFERÊNCIAS}

BELEDELLI, S.; SANTOLIN, M. Aplicação dos dez passos da alimentação saudável e dez passos da alimentação adequada e saudável e avaliação antropométrica em praticantes de pilates em uma academia do norte do Rio Grande do Sul. Revista Brasileira de Nutrição Esportiva, São Paulo, v. 11, n. 67, suplementar 1, p. 910-915. jan./dez. 2017.

BOOG, M. C. F. Educação em nutrição: integrando experiências. 1. ed. Campinas, SP: Komedi, 2013. p. 286.

BRASIL. Ministério da Saúde MS. Política Nacional de Promoção da Saúde. Brasília, DF: MS, 2006.

BRASIL. Ministério da Saúde. Secretaria de Atenção à Saúde. Coordenação-Geral de Alimentação e Nutrição. Política Nacional de Alimentação e Nutrição. Brasília, DF: Ministério da Saúde, 2012a.

BRASIL. Ministério do Desenvolvimento Social e Combate à Fome. Secretaria Nacional de Segurança Alimentar Nutricional. Marco de Referência de Educação Alimentar e Nutricional Para as Políticas Públicas. Brasília, DF: Ministério da Saúde, 2012b.

BRASIL. Câmara Interministerial de Segurança Alimentar e Nutricional. Estratégia intersetorial de prevenção e controle da obesidade: recomendações para Estados e municípios. Brasília, DF: Caisan, 2014a.

BRASIL. Ministério do Desenvolvimento Social e Combate à Fome. Educação Alimentar e Nutricional: uma estratégia para promoção do direito humano à alimentação adequada. Brasília: MDS, 2014b.

BRASIL. Ministério da Saúde; Secretaria da Atenção à Saúde; Departamento de Atenção Básica. Guia alimentar para a população brasileira. 2. ed. Brasília: Ministério da Saúde, 2014c. 
BRASIL. Decreto № 8.553, de 3 de novembro de 2015. Institui o Pacto Nacional para a Alimentação Saudável. Diário Oficial da União, Brasília, DF, 4 nov. 2015.

BRASIL. Ministério da Saúde. Vigilância de fatores de risco e proteção para doenças crônicas por inquérito telefônico. Brasília: Ministério da Saúde; Agência Nacional de Saúde Suplementar; 2018.

BRITO, P. D. et al. Educação alimentar e nutricional para o controle de comorbidades em pessoas com doenças infecciosas. Revista Brasileira de Promoção em Saúde, Fortaleza, v. 30, p. 141-148, jan./mar. 2017.

CERVATO-MANCUSO, A. M. et al. Educação alimentar e nutricional como prática de intervenção: reflexão e possibilidades de fortalecimento. Physis: Revista de Saúde Coletiva, v. 26, p. 225-249, 2016.

CERVATO-MANCUSO, A. M. Elaboração de Programa de Educação Nutricional. In: GARCIA, Rosa Wanda Diez; MANCUSO, Ana Maria Cervato (coord.). Nutrição e metabolismo: mudanças alimentares e educação nutricional. Rio de Janeiro: Guanabara Koogan, 2013. p. 187-197.

DE MELO, M. P. F. et al. Núcleo de Apoio à Saúde da Família - Nasf: Práticas de um acadêmico de nutrição. Revista Eletrônica de Extensão, v. 13, n. 24, 2016.

DEUS, R. M. et al. Impacto de intervenção nutricional sobre o perfil alimentar e antropométrico de usuárias do Programa academia da saúde. Ciência \& Saúde Coletiva, Rio de Janeiro, v. 20, n. 6, p. 1.937-1.946, jun. 2015.

DO NASCIMENTO, R. P. et al. Relato de experiência: educação alimentar e nutricional com pacientes renais crônicos em hemodiálise. Revista Nutrição Clínica Die. Hosp., v. 36, n. 3, p. 31-37, 2016.

ESPERANÇA, L. M. B., GALISA, M. S. Programa de educação alimentar e nutricional: diagnóstico, objetivos, conteúdo e avaliação. In: GALISA, M. S. et al. Educação alimentar e nutricional: da teoria à prática. Vila Mariana, SP: Roca, 2014.

FAGIOLI, D.; NASSER, L. Educação nutricional na infância e na adolescência: planejamento, intervenção, avaliação e dinâmicas. São Paulo: RCN Editora, 2006.

FRANÇA C. J.; CARVALHO, V. C. H. dos S. de. Estratégias de educação alimentar e nutricional na Atenção Primária à Saúde: uma revisão de literatura. Saúde Debate, Rio de Janeiro, v. 41, n. 114, p. 932-948, jul-set, 2017.

FREIRE, P. Pedagogia da Autonomia. São Paulo: Paz e Terra, 2004.

FROIS, S. F. B.; DOURADO, L. M.; PINHO, L. Promoção da alimentação saudável entre profissionais de saúde na atenção primária. Revista Brasileira de Promoção da Saúde, Fortaleza, v. 29, p. 621-626, out./dez. 2016.

GOMES, A. C. M. et al. Impacto de estratégias de educação nutricional sobre variáveis antropométricas e conhecimento alimentar. Revista Brasileira de Promoção em Saúde, Fortaleza, v. 26, n. 4, p. 462-469, out./ dez. 2013.

IBGE. Instituto Brasileiro de Geografia e Estatística. Pesquisa Nacional por Amostra de Domicílios 2010. Rio de Janeiro: IBGE, 2010.

MACHADO, J. C. et al. Análise de três estratégias de educação em saúde para portadores de hipertensão arterial. Ciência \& Saúde Coletiva, Rio de Janeiro, v. 12, n. 2, p. 611-620, fev. 2016.

PEREIRA M. A. et al. Desafios e reflexões na implantação de um programa de Educação Alimentar e Nutricional (EAN) em indivíduos com excesso de peso. Rev. Bras. Promoção Saúde, Fortaleza, v. 28, p. 290-296, abr./jun. 2015.

RAMOS, M.; SCARPARO, A. L. S. Proposta metodológica para o planejamento de programa de educação alimentar e nutricional de acordo com os pressupostos de Paulo Freire. SEMINÁRIO NACIONAL: DIÁLOGOS PAULO FREIRE, 10., Democracia, sujeitos coletivos e a Pedagogia da Esperança, 2016. Porto Alegre. Anais [...]. Porto Alegre, RS, 2016.

ROSA, P. B. Z.; GIUSTI, L., RAMOS, M. Educação alimentar e nutricional com universitários residentes de moradia estudantil. Revista Ciência e Saúde, v. 9, n. 1, p. 15-20 jan./abr. 2016.

RIBEIRO, H.; JAIME, P. C.; VENTURA, D. Alimentação e sustentabilidade. Estudos Avançados, São Paulo, v. 31, n. 89, p. 185-198, abr. 2017.

SANTOS, L. A. S. O fazer educação alimentar e nutricional: algumas contribuições para reflexão. Ciência \& Saúde Coletiva, Rio de Janeiro, v. 17, n. 2, p. 453-462, fev. 2012.

SCARPARO, A. L. S. Crenças sobre o ensino da temática alimentação saudável no ambiente escolar. 2017. Tese (Doutorado) - Universidade Federal do Rio Grande do Sul - UFRGS, Programa de Pós-Graduação em Educação em Ciências: Química da Vida e Saúde, Porto Alegre, RS, 2017.

SILVA, A. C. S. Promoção do autocuidado de idosos para o envelhecer saudável: aplicação da Teoria de Nola Pender. Texto Contexto Enferm., Florianópolis, v. 19, p. 745-53, out./dez. 2010. 